\title{
DUALITY FOR FRACTIONAL MINIMAX PROGRAMMING PROBLEMS
}

\author{
SHRI RAM YADAV ${ }^{1}$ AND R. N. MUKHERJEE ${ }^{1}$
}

(Received 2 November 1987; revised 12 January 1989)

\begin{abstract}
Duality theory is discussed for fractional minimax programming problems. Two dual problems are proposed for the minimax fractional problem: minimize $\max _{y \in Y} f(x, y) / h(x, y)$, subject to $g(x) \leq 0$. For each dual problem a duality theorm is established. Mainly these are generalisations of the results of Tanimoto [14] for minimax fractional programming problems. It is noteworthy here that these problems are intimately related to a class of nondifferentiable fractional programming problems.
\end{abstract}

\section{Introduction}

Duality Theory for convex differentiable programs has drawn considerable attention from the workers in the field of mathematical programming so far. A comprehensive account of the relevant results in this area can be found in Craven [2]. In particular the reference [2] also gives a useful account of the treatment of Lagrangian and Wolfe duals for differentiable programs and further in the cited reference some applications of the Lagrangian theory are also given. Another duality concept known as apex dual which is different from that of the Lagrangian Dual has been developed by Duffin [3] and Duffin, Karney and Prisman [4] in the case of primal constraints. B. Mond [7] has treated nondifferentiable mathematical programming problem in detail and further Tanimoto [14] develped a duality theory for each class of problems. In the present work our primary objective is to develop a duality theory for fractional min-max programming which eventually is more general than

\footnotetext{
${ }^{1}$ Department of Applied Mathematics, Institute of Technology, Banaras Hindu University, Varanasi-221 005, India.

(C) Copyright Australian Mathematical Society 1990, Serial-fee code 0334-2700/90
} 
ordinary fractional programming. Although our results basically extend the results of Tanimoto, at the same time they also extend the dual theorems such as those obtained for min-max objective function involving basic functions of the type $f_{i} / g_{i}, 1 \leq i \leq p$ in the work of Jagannathan and Schaible [5], where the functions $f_{i}$ and $g_{i}$ were nondifferentiable.

In fact in [13] Singh has established necessary and sufficient optimality conditions for min-max type of Fractional programming as treated in the present paper but duality theory for such problems do not appear in his treatment. Our results also differ essentially from the duality results obtained in S. Chandra, B. D. Craven and B. Mond [1] in their generality. In the current literature there are dual theorems for Multi-objective programming problems involving a vector minimization of $n$-tuple of functions which are either differentiable or subdifferentiable. For a preliminary account of such results one can refer to the work of Sawaragi, Nakayama and Tanino [10]. Our contention is that the dual theorems of the type developed in the present work can be extended to the setting of vector optimization problem where each entry of the $n$-tuple of functions involves a min-max type of objective function separately.

A point to be made with regard to the essential feature of the subject is that the duality theory for convex programs is based on a concept of the 'adjoint' of a convex bifunction (see Rockafellar [9]). the adjoint operation for bifunctions may be regarded as a generalization of the adjoint operation for linear transformations. Also one can build a convex algebra parallel to linear algebra in the gamut of the Duality theory.

Our objective function treated here is as follows

$$
F(x)=\operatorname{Sup}_{y \in Y} \frac{f(x, y)}{h(x, y)}
$$

where $Y$ is a compact subset of $R^{m}, f(.,):. R^{n} \times R^{m} \rightarrow R$ is a differentiable function on $R^{n} \times R^{m}$ and $h(.,):. R^{n} \times R^{m} \rightarrow R$ is also a differentialbe function on $R^{n} \times R^{m}$. Throughout this paper we assume that $h(x, y)>0$ for each $(x, y)$ in $X \times Y$, where $X$ is a set of feasible solutions of the problem. Also we assume that $f(., y)$ and $-h(., y)$ are convex functions of $x$ for each $y$. Notice that although $f$ and $h$ are differentiable functions $F($.) may not be differentiable. Also $F($.) need not be a convex function.

The primal problem is as follows:

Problem (P) Minimize $F(x)$

Subject to $x \in X=\left\{x \in R^{n} \mid g(x) \leq 0\right\}$,

where $F$ is the function defined by (1) and $g():. R^{n} \rightarrow R^{p}$ is a convex and differentiable function. We assume that $X$ is nonempty. In this paper our aim is to formulate two dual problems to primal problem (P) and then to 
establish duality relationship. We obtain a duality theorem for each dual problem and converse duality is also discussed.

Throughout the paper, it is assumed that $f(., y)$ and $-h(., y)$ are convex functions of $x$ for each $y . \nabla_{x} f,-\nabla_{x} h$ and $\nabla_{x} g$ denote the gradient vectors of $f(., y),-h(., y)$ and $g($.$) , respectively. Given a real valued function F$ on $R^{n}, \nabla^{2} F$ denotes the $n \times n$ Hessian matrix.

In the development of the matter what follows in the ensuiñ̄ paragrapiph we have adopted the essential techniques of [14] which are necessary to make the treatment as coherent as possible.

\section{Duality theorem}

Let $X$, the set of feasible solution be a compact set. For each $x \in X$, we define,

$$
\begin{aligned}
I(x) & =\left\{i: g_{i}(x)=0\right\}, \\
Y(x) & =\left\{y \in Y ; f(x, y) / h(x, y)=\operatorname{Sup}_{z \in Y} f(x, z) / h(x, z)\right\},
\end{aligned}
$$

$K=$ the set of triplets $(s, t, \bar{y})$, where $s$ ranges over the positive integers such that $1 \leq s \leq n+1 ; t=\left(t_{1}, \ldots, t_{s}\right)$ an $s$-dimensional vector with $t \geq 0$, $\sum_{i=1}^{s} t_{i}=1 ; y=\left(y_{1}, \ldots, y_{s}\right)$ an $m s$-dimensional vector with $y_{i} \in Y(x)(i=$ $1, \ldots, s)$ for some $x \in R^{n}$.

For $x \in X,(s, t, \bar{y}) \in K, y_{i} \in Y(x), \quad v=f\left(x, y_{i}\right) / h\left(x, y_{i}\right)$.

Also we define

$$
H(s, t, \bar{y})=\left\{(x, \mu) \in R^{n} \times R^{p} \mid x \text { and } \mu \text { satisfying }\left\{y_{1}, \ldots, y_{s}\right\} \subset Y(x)\right.
$$

and

$$
\begin{aligned}
\left\{\sum_{i=1}^{s}\left(t_{i} / h\left(x, y_{i}\right)\right)\left(\nabla_{x} f\left(x, y_{i}\right)\right)-v \nabla_{x} h\left(x, y_{i}\right)+\sum_{i=1}^{p} \mu_{i} \nabla g_{i}(x)=0,\right. & \\
\mu & \left.=\left(\mu_{1}, \ldots, \mu_{p}\right) \geq 0\right\} .
\end{aligned}
$$

Now we define a dual problem as follows:

Problem (D)

$$
\underset{(s, t, \bar{y}) \in K}{\operatorname{Maximize}} \operatorname{Sup}_{(x, \mu) \in H(s, t, \bar{y})} F(x)+\sum \mu_{i} g_{i}(x) .
$$

If for a triplet $(s, t, \bar{y})$ in $K$, the set $H(s, t, \bar{y})$ is empty we define the supremum over it to be $-\infty$. Under the convexity condition imposed on the functions $f,-h$ and $g$, the next theorem state a duality relationship between problem (P) and (D). 
THEOREM 2.1. Let $x^{*} \in X$ be an optimal solution of the problem (P) and $\nabla g_{i}\left(x^{*}\right)\left(i \in I\left(x,{ }^{*}\right)\right)$ be linearly independent. Then there exist $\left(s^{*}, t^{*}, \bar{y}^{*}\right)$ in $K$ and $\mu^{*} \in R^{p}, \mu^{*} \geq 0$, with $\left(x^{*}, \mu^{*}\right) \in H\left(s^{*}, t^{*}, \bar{y}^{*}\right)$ such that $\left(s^{*}, t^{*}, \bar{y}^{*}\right)$ and $\left(x^{*}, \mu^{*}\right)$ give an optimal solution to problem (D). Also, the two problems (P) and (D) have the same extremal values.

Proof. Since $x^{*}$ is an optimal solution of $(\mathrm{P})$ and $\nabla g_{i}\left(x^{*}\right)\left(i \in I\left(* x^{*}\right)\right)$ are linearly independent, therefore by Schmitendorf's Theorem 1 [12] and its corollary, there exist a positive integer $s^{*}, 1 \leq s^{*} \leq n+1, t^{0}=\left(t_{1}^{0}, \ldots, t_{s}^{0}\right)$ with $t_{i}^{0}>0\left(i=1, \ldots, s^{*}\right), \bar{y}^{*}=\left(y_{1}^{*}, \ldots, y_{s}^{*}\right)$ with $y_{i}^{*} \in Y\left(x^{*}\right)(i=1, \ldots, s)$ and $\mu^{0}=\left(\mu_{1}^{0}, \ldots, \mu_{p}^{0}\right)$ with $\mu_{i}^{0} \geq 0(i=1, \ldots, p)$ such that

$$
\begin{gathered}
\sum_{i=1}^{s^{*}}\left(t_{i}^{0} / h\left(x^{*}, y_{i}^{*}\right)\left(\nabla_{x} f\left(x^{*}, y_{i}^{*}\right)\right)-\left(t_{i}^{0} / h\left(x^{*}, y_{i}^{*}\right)^{2}\right)\left(f\left(x^{*}, y_{i}^{*}\right) \nabla_{x} h\left(x^{*}, y_{i}^{*}\right)\right)\right. \\
+\sum_{i=1}^{p} \mu_{i}^{0} \nabla g_{i}\left(x^{*}\right)=0,
\end{gathered}
$$

or

$$
\begin{gathered}
\sum_{i=1}^{s^{*}}\left(t_{i}^{0} / h\left(x^{*}, y_{i}^{*}\right)\right)\left(\nabla_{x} f\left(x^{*}, y_{i}^{*}\right)-v^{*} \nabla_{x} h\left(x^{*}, y_{i}^{*}\right)\right)+\sum_{i=1}^{p} \mu_{i}^{0} \nabla g_{i}\left(x^{*}\right)=0, \\
\mu_{i}^{0} g_{i}\left(x^{*}\right)=0, i=1, \ldots p .
\end{gathered}
$$

Let $\alpha=\sum_{i=1}^{s^{*}} t_{1}^{0}$, then $\left(s^{*}, \alpha^{-1} t^{0}\right)$ belongs to $K$ and $\left(x^{*}, \alpha^{-1} \mu^{0}\right)$ belongs to $H\left(s^{*}, \alpha^{-1} t^{0}, \bar{y}^{*}\right)$. Put $t^{*}=\alpha^{-1} t^{0}$ and $\mu^{*}=\alpha^{-1} \mu^{0}$. We first show that $\left(x^{*}, \mu^{*}\right)$ attains the maximum of the following problem:

$$
\begin{aligned}
& \text { Maximize } F(x)+\sum_{i=1}^{p} \mu_{i} g_{i}(x) \\
& \text { Subject to }(x, \mu) \in H\left(s^{*}, t^{*}, y^{*}\right) .
\end{aligned}
$$

For any $(x, \mu) \in H\left(s^{*}, t^{*}, \bar{y}^{*}\right)$, using $\left\{y_{1}^{*}, \ldots, y_{s^{*}}^{*}\right\} \subset(x)$ and the convexity of $f,-h, g$ and remarking $\mu^{*} g_{i}\left(x^{*}\right)=0(i=1, \ldots, p)$, we have

$$
\begin{aligned}
F\left(x^{*}\right) & +\sum_{i=1}^{p} \mu_{i}^{*} g_{i}\left(x^{*}\right)-\left\{F(x)+\sum_{i=1}^{p} \mu_{i} g_{i}(x)\right\} \\
= & \sum_{i=1}^{s^{*}} t_{i}^{*}\left(f\left(x^{*}, y_{i}^{*}\right) / h\left(x^{*}, y_{i}^{*}\right)\right)+\sum_{i=1}^{p} \mu_{i}^{*} g_{i}\left(x^{*}\right) \\
& -\left\{\sum_{i=1}^{s^{*}} t_{i}^{*}\left(f\left(x, y_{i}^{*}\right) / h\left(x, y_{i}^{*}\right)\right)+\sum_{i=1}^{p} \mu_{i} g_{i}(x)\right\}
\end{aligned}
$$

(continues) 


$$
\begin{aligned}
\geq & \sum_{i=1}^{s^{*}}\left(t_{i}^{*} / h\left(x, y_{i}^{*}\right)\right)\left(\nabla_{x} f\left(x, y_{i}^{*}\right)-v^{*} \nabla_{x} h\left(x, y_{i}^{*}\right)\right)\left(x^{*}-x\right)-\sum_{i=1}^{p} \mu_{i} g_{i}(x) \\
\geq & \left\{\sum_{i=1}^{s^{*}}\left(t_{i}^{*} / h\left(x, y_{i}^{*}\right)\right)\left(\nabla_{x} f\left(x, y_{i}^{*}\right)-v^{*} \nabla_{x} h\left(x, y_{i}^{*}\right)\right)+\sum_{i=1}^{p} \mu_{i} \nabla g_{i}(x)\right\} \\
& \times\left(x^{*}-x\right)-\sum_{i=1}^{p} \mu_{i} g_{i}\left(x^{*}\right) \\
\geq & 0 .
\end{aligned}
$$

In the last inequality, $(x, \mu) \in H\left(s^{*}, t^{*}, \bar{y}^{*}\right)$ and $g_{i}\left(x^{*}\right) \leq 0(i=1, \ldots, p)$ are used. Hence we obtain

$$
F\left(x^{*}\right)=F\left(x^{*}\right)+\sum_{i=1}^{p} \mu_{i}^{*} g_{i}\left(x^{*}\right) \geq F(x)+\sum_{i=1}^{P} \mu_{i} g_{i}(x)
$$

for all $(x, \mu) \in H\left(s^{*}, t^{*}, \bar{y}^{*}\right)$. Further, to complete the proof, we must show that for any $(s, t, \bar{y})$ in $K$,

$$
\operatorname{Sup}_{(x, \mu) \in H(s, t, \bar{y})} F(x)+\sum_{i=1}^{p} \mu_{i} g_{i}(x) \leq F\left(x^{*}\right) .
$$

We may assume that $H(s, t, \bar{y})$ is nonempty. Take any $(x, \mu)$ from $H(s, t, \bar{y})$. By using

$$
\begin{gathered}
F(x)=\sum_{i=1}^{s} t_{i}\left(f\left(x, y_{i}\right) / h\left(x, y_{i}\right)\right), \\
F\left(x^{*}\right) \geq \sum_{i=1}^{s} t_{i}\left(f\left(x^{*}, y_{i}\right) / h\left(x^{*}, y_{i}\right)\right)
\end{gathered}
$$

and

$$
\sum_{i=1}^{p} \mu_{i} g_{i}\left(x^{*}\right) \leq 0
$$

we have

$$
\begin{aligned}
F(x) & +\sum_{i=1}^{p} \mu_{i} g_{i}(x)-F\left(x^{*}\right) \\
\leq & \sum_{i=1}^{s} t_{i}\left(f\left(x, y_{i}\right) / h\left(x, y_{i}\right)\right)+\sum_{i=1}^{p} \mu_{i} g_{i}(x) \\
& -\sum_{i=1}^{s} t_{i}\left(f\left(x^{*}, y_{i}\right) / h\left(x^{*}, y_{i}\right)\right)-\sum_{i=1}^{p} \mu_{i} g_{i}\left(x^{*}\right)
\end{aligned}
$$




$$
\begin{aligned}
\leq & \left\{\sum_{i=1}^{s}\left(t_{i} / h\left(x, y_{i}\right)\right)\left(\nabla_{x} f\left(x, y_{i}\right)-v \nabla_{x} h\left(x, y_{i}\right)\right)+\sum_{i=1}^{p} \mu_{i} \nabla g_{i}(x)\right\} \\
& \times\left(x^{*}-x\right)=0 .
\end{aligned}
$$

Since $(x, \mu)$ belongs to $H(x, t, \bar{y})$. Thus (3) is proved from which follows the first assertion of this theorem and (2) implies the second assertion, because $F\left(x^{*}\right)$ is the extremal value of Problem (P).

\section{Alternative duality theorem}

As the special case of the programming problems considered, we let here,

$$
f(x, y) / h(x, y)=k(x) / q(x)+x^{t} y,
$$

where $t$ denote the transpose and $k(\dot{)},-q(\dot{)}$ are convex and differentiable functions defined on $R^{n}$. Putting $h(x, y)=1$ and $q(x)=1$, we can obtain the problem studied in $[11,13]$. Notice that in this case the set $K$ can be represented by the set of elements $\omega$ in $Y$ such that $x^{t} \omega=\operatorname{Sup}_{z \in Y} x^{t} z$ for some $x$ of $X$.

In view of a dual problem [Problem (D)] introduced by Tanimoto [14, Section 2], we are interested here also to introduce a less restrictive dual problem as follows:

Problem (D*)

$$
\operatorname{Maximize}_{(s, t, \bar{y}) \in \bar{K}} \operatorname{Sup}_{(x, \mu) \in \bar{H}(s, t, \bar{y})} \sum_{i=1}^{s} t_{i} f\left(x, y_{i}\right) / h\left(x, y_{i}\right)+\sum_{i=1}^{p} \mu_{i} g_{i}(x) .
$$

Here $\bar{K}$ denotes the set of triplets $(s, t, \bar{y})$, where $s$ ranges over the integers $1 \leq s \leq n+1, t=\left(t_{1}, \ldots, t_{s}\right), t_{i} \geq 0(i=1, \ldots, s)$, with $\sum_{i=1}^{s} t_{i}=1, \bar{y}=$ $\left(y_{1}, \ldots, y_{s}\right)$ with $y_{i} \in Y$ for all $i=1, \ldots, s$ and we define

$$
\begin{aligned}
\bar{H}^{*}(s, t, \bar{y})=\left\{(x, \mu) \in R^{n} \times R^{p} \mid\right. & \sum_{i=1}^{s}\left(t_{i} / h(x, y)\right)\left(\nabla_{x} f(x, y)\right. \\
& \left.-v \nabla h(x, y))+\sum_{i=1}^{p} \mu_{i} \nabla g_{i}(x)=0, \mu \geq 0\right\} .
\end{aligned}
$$

Note that the condition, $\left\{y_{i}, \ldots y_{s}\right\} \subset Y(x)$ for some $x \in X$, is droped in this dual problem. Now our objective is to relate problem $(P)$ to $\left(D^{*}\right)$ rather than to (D). 
To do this we consider the following primal and dual problems for each $\eta=(s, t, \bar{y}) \in \bar{K}$ :

$$
\begin{aligned}
& \text { Problem }\left(P_{\eta}\right) \quad \text { Maximize } \sum_{i=1}^{s} t_{i}\left(f\left(x, y_{i}\right) / h\left(x, y_{i}\right)\right) \\
& \text { Subject to } x \in X=\{x \mid g(x) \leq 0\} \\
& \text { Problem }\left(D_{\eta}\right) \quad \text { Minimize } \sum_{i=1}^{s} t_{i}\left(f\left(x, y_{i}\right) / h\left(x, y_{i}\right)\right)+\sum_{i=1}^{p} \mu_{i} g_{i}(x) \\
& \text { Subject to }(x, \mu) \in H^{*}(s, t, \bar{y}) .
\end{aligned}
$$

These are as usual (differentiable but not convex) fractional programming problem and its dual problem. This can be also convexified by using the Manas and Schaible transformation. Now we denote the extremal values of Problems $\left(\mathrm{P}_{\eta}\right)$ and $\left(\mathrm{D}_{\eta}\right)$ by $\left(\mathrm{P}_{e}\right)$ and $\left(\mathrm{D}_{e}\right)$ respectively and those of Problems $(P),(D)$ and $\left(D^{*}\right)$ by $\min (P), \max (D)$ and $\max \left(D^{*}\right)$, respectively. The next theorem given below relates Problems $(P)$ to $\left(D^{*}\right)$.

THEOREM 3.1. If $x^{*}$ is optimal for $(\mathrm{P})$ and $\nabla g_{i}\left(x^{*}\right)\left(i \in I\left(x^{*}\right)\right)$ are linearly independent, then there exist $\left(s^{*}, t^{*}, y^{*}\right)$ in $K^{*}$ and $\mu^{*} \in R^{p}, \mu^{*} \geq 0$, with $\left(x^{*}, \mu^{*}\right) \in H^{*}\left(s^{*}, t^{*}, \bar{y}^{*}\right)$ such that $\left(s^{*}, t^{*}, \bar{y}^{*}\right)$ and $\left(x^{*}, \mu^{*}\right)$ give an optimal solution to $\left(\mathrm{D}^{*}\right)$. Furthermore the two problems $(\mathrm{P})$ and $\left(\mathrm{D}^{*}\right)$ have the same extremal values.

The proof of Theorem 3.1 can be given on similar lines as that of Theorem 2.1.

\section{Converse duality}

In this section we assume our fractional function as follows:

$$
(f(x, y) / h(x, y))=k(x) / q(x)+y^{t}(r(x) / q(x)) .
$$

Now using the usual Manas and Schaible transformation, the above function can be expressed as follows:

$$
\psi\left(z_{0}, z, y\right)=z_{0} k\left(z / z_{0}\right)+y^{t} z_{0} r\left(z / z_{0}\right)
$$

or

$$
\psi\left(z_{0}, z, y\right)=\alpha\left(z_{0}, z\right)+y^{t} \beta\left(z_{0}, z\right)
$$


which is very similar to the function adopted in [14] Section 4. Here also $\alpha\left(z_{0}, z\right)$ and $\beta\left(z_{0}, z\right)$ are convex and twice continuously differentiable functions from $R^{n}$ and $R^{m}$ respectively. The primal problem is as follows:

Problem $\overline{(\mathrm{P})} \quad$ Minimize $F(x)=\max _{y \in Y} z_{0} k\left(z / z_{0}\right)+y^{t} z_{0} r\left(z / z_{0}\right)$

Subject to $g\left(z / z_{0}\right) \leq 0$,

where $g$ is twice continuously differentiable. Let us define compact constraint $Y$ as follows:

$$
Y=\left\{y \in R^{m} \mid \omega(y) \leq 0\right\},
$$

where $\omega(): R^{m} \longrightarrow R^{q}$ is a convex and differentiable function. We assume that $Y \subset\{y \mid y \geq 0\}$.

We shall say that following condition (*) is satisfied at $y^{0} \in Y$ if

$$
c \in R^{q}, \nabla \omega\left(y^{0}\right) c=0, c^{t} \omega\left(y^{0}\right)=0, c \geq 0 \text { imply } c=0,
$$

Since $Y$ is convex, it is easily seen that in this case problem $\left(D^{*}\right)$ is:

$$
\begin{aligned}
& \text { Maximize } z_{0} k\left(z / z_{0}\right)+y^{t} z_{0} r\left(z / z_{0}\right) \\
& \text { Subject to } \nabla\left(z_{0} k\left(z / z_{0}\right)\right)+y^{t} \nabla\left(z_{0} r\left(z / z_{0}\right)\right) \\
& +\mu^{t} \nabla g\left(z / z_{0}\right)=0 \\
& \mu \geq 0, \quad \omega(y) \geq 0 .
\end{aligned}
$$

Now, to prove the converse duality theorem between $(\mathbf{P})$ and its dual problem, we state the following Lemma as a special case of Lemma 1 of Tanimoto [14].

LEMMA 1. Let $\left(z_{0}^{*}, z^{*}, y^{*}, \mu^{*}\right)$ be optimal for (D)* and let the matrix $\nabla^{2}\left(z_{0}^{*} k\left(z^{*} / z_{0}^{*}\right)\right)+\nabla^{2} y^{* t}\left(z_{0}^{*} r\left(z^{*} / z_{0}^{*}\right)\right)+\nabla^{2} \mu^{* t} g\left(z^{*} / z_{0}^{*}\right)$ be nonsingular. Suppose the condition (*) is satisfied at $y^{*}$. Then $y^{*}$ belongs to $Y\left(z^{*} / z_{0}^{*}\right)$.

We omit the proof here, because this can be proved very easily following the proof of Lemma 1 of [14].

TheOREM 4.1. (Converse duality) Let $\left(z_{0}^{*}, z^{*}, y^{*}, \mu^{*}\right)$ be as in Lemma 1 , then $\left(z_{0}^{*}, z^{*}\right)$ is optimal for $(\overline{\mathrm{P}})$ and the two extremal values of Problem $(\overline{\mathrm{P}})$ and (D)* are equal.

The above results can be proved in a similar manner as in the case of Theorem 3 of [14].

\section{Acknowledgements}

The authors are thankful to the referees for their valuable comments. The work was supported by C.S.I.R., S.R.F. Grant No. 9/13/135/85-EMR-1. 


\section{References}

[1] S. Chandra, B. D. Craven, and B. Mond, "Generalized fractional programming duality: a ratio game approach," J. Austral. Math. Soc. Ser. B 28 (1986) 181-210.

[2] B. D. Craven, Mathematical Programming and Control Theory (Chapman and Hall, London, 1978).

[3] R. J. Duffin, "Numerical estimation of optima by use of dual inequalities", in Semi-infinite programming and applications (eds. A. V. Fiacco and K. O. Knrtanek), (Springer-Verlag, Berlin, 1983) 118-127.

[4] R. J. Duffin, D. F. Karney and E. Z. Prisman, "Apex duality for constrained optimization”, J. Austral. Math. Soc. Ser. B 28 (1986) 134-146.

[5] R. Jagannathan and S. Schaible, “Duality in generalized fractional programming via Farkas' lemma", J. of Opt. Th. and Appl. 4 (1983) 417-424.

[6] S. Kaur, "Subgradient duality in fractional programming", Indian J. of Pure and Applied Math. 13 (3) (1982) 287-298.

[7] B. Mond, "A class of nondifferentiable mathematical programming problems, J. of Math. Anal. and Appl. 46 (1974) 169-174.

[8] O. L. Mangasarian, Nonlinear programming (McGraw Hill, New York, 1969).

[9] R. Rockafellar, Convex analysis (Princeton University, press, Princeton, New Jersey, 1970).

[10] Y. Sawaragi, H. Nakayama, and T. Tanino, Theory of multiobjective optimization (Academic Press, New York, 1985).

[11] M. Schechter, "A subgradient duality theorem", J. of Math. Anal. and Appl. 61 (1977) 850-855.

[12] W. E. Schmitendorf, "Necessary conditions and sufficient conditions for static minimax problems", J. of Math. Anal. and Appl. 57 (1977) 683-693.

[13] C. Singh, "Optimality conditions for fractional minimax programming, J. of Math. Anal. and Appl. 100 (1984) 409-415.

[14] S. Tanimoto, "Duality for a class of nondifferentiable mathematical programming problems", J. of Math. Anal. and Appl. 79 (1981) 283-294.

[15] P. Wolf, "A duality theorem for nonlinear programming", Quart. J. of Appl. Math. 19 (1961) 239-244. 